\title{
URINARY TRACT DUPLICATION IN CHILDHOOD
}

BY

\author{
J. H. JOHNSTON \\ From Alder Hey Hospital, Liverpool
}

(RECEIVED FOR PUBLICATION OCTOBER 10, 1960)

\begin{abstract}
The incidence of duplication of the urinary tract has variously been estimated by different authors to be between the extremes of $0.6 \%$ (Campbell, 1951) and $6 \%$ (Thompson and Amar, 1958) of individuals. The anomaly may remain symptomless and undetected throughout life, but it carries a predisposition to pathological complications, the most severe and urgent of which occur in early childhood, at which age ureteric duplication is not infrequently a urological emergency.
\end{abstract}

\section{Embryology}

Ureteric duplication follows the formation, at about the fifth week of intrauterine life, of an accessory ureteric bud the precise site of origin of which determines the ultimate anatomical features. If the bud arises from the main ureter the final result is an incomplete duplication with the ureters uniting above the bladder; if it takes origin from the mesonephric duct immediately cranial to the main ureter a complete duplication is produced with both ureters opening separately into the bladder. Since the lower end of the mesonephric duct becomes incorporated in the wall of the urogenital sinus the accessory ureter is carried caudally, crossing the main one, and its orifice is found, as a rule, below and medial to the main orifice (Weigert-Meyer law). When the accessory bud arises from the mesonephric duct at a still more cranial site it is carried farther caudally and its orifice comes to lie outside the bladder in that portion of the urogenital sinus which incorporates the mesonephric duct or in the derivatives of the duct itself. Thus, in the male, the accessory ureter may enter the prostatic urethra above the ejaculatory duct or open into the duct, the seminal vesicle or the vas deferens. In the female the orifice may lie in the urethra or in the vestibule adjacent to the urethral meatus; less commonly secondary fistulous communication with the vagina or the uterus, possibly through Gartner's duct, may develop during prenatal life. The accessory ureter most commonly arises from the cranial aspect of the main ureter or of the mesonephric duct and thus makes contact with the cranial portion of the metanephrogenic blastema. As a result, the accessory ureter, the upper extremity of which dilates to form a pelvis without any obvious pelvi-ureteric junction, drains by one, two or three calyces usually the upper third or less of the double kidney. The exterior of the kidney may show no evidence of duplicity or a groove may indicate the division between the two portions. The pelvicalyceal systems of each part are, as a rule, entirely separate; communication between the two has, however, been described by Beer and Mencher (1938) and by Braasch (1912). Very uncommonly the accessory ureter takes origin from the caudal aspect of its fellow or of the mesonephric duct and so drains the lower portion of the double kidney.

\section{Material}

The present review is based on a clinical series of 73 children with complete or incomplete duplication and of nine cases in which the abnormality was a post-mortem finding. Cases of the minimal degree of duplication, e.g. the bifid pelvis, have been excluded and so too have those in which a supernumerary ureter terminated outside the urinary tract or in the lower urethra in the female, i.e. the so-called ectopic ureter, since in this group the symptomatology and the diagnostic problems are quite different. Analysis of the series supports the findings of other authors that duplication, at least as a clinical problem, is commoner in the female than in the male $(57: 25)$, that it is more commonly unilateral than bilateral $(63: 19)$, that in unilateral cases each side is more or less equally frequently affected (right 35; left 28) and that in bilateral cases the anatomical pattern is not necessarily symmetrical. Triple ureters were found in two patients and quadruple ureters in one. Associated but unrelated congenital abnormalities of the urinary tract were present in three patients with, respectively, a horseshoe kidney, a large vesical diverticulum of congenital type and urethral valves. Although ureteric duplication is initiated at an early phase of embryonic life the responsible stimulus appears, in most cases, to act locally since obvious extra-urinary congenital 
abnormalities were not present in any patient in the present clinical series. Complicated or uncomplicated duplication was found at operation in one patient with exomphalos and at necropsy in six infants with other gross anomalies such as oesophageal atresia, imperforate anus, achondroplasia, myelomeningocele, diaphragmatic hernia and congenital heart disease. In 15 cases the urinary tract duplication was considered to be purely coincidental and unrelated to the patient's symptoms. These children were, for the most part, enuretics or cases of obscure abdominal pain in whom an abdominal radiograph, by showing a large renal shadow, led to intravenous pyelography. Five patients complained of recurrent loin pain on the side of an uncomplicated, uninfected ureteric duplication.

\section{Complications}

The main childhood complications of double ureters which are of clinical importance fall into two groups: (1) anatomical lesions consisting of obstructive or non-obstructive dilatation of one or both pyelons, associated with impairment of renal function, urinary stasis and infection; (2) chronic or recurrent urinary infection with a reduplicated but otherwise anatomically normal urinary tract. Patients with structural complications usually present at a much earlier age than those with infection alone. In this retrospective review of cases it was, in some instances, impossible to determine with certainty the precise pathologico-anatomical lesion which was present because of incomplete investigation before excisional surgery, but it has been possible, excluding the indeterminate cases, to recognize certain specific types of pyelonic dilatation, some of which are peculiar to the duplicated urinary tract and some which may also affect the single ureter. In several cases different lesions were present in the same patient.

The Obstructed Megaureter associated with Ectopic Ureterocele. Ectopic ureterocele (Fig. 1), with obstruction of the corresponding accessory pyelon leading to its dilatation and to ureteric tortuosity, was found in 11 instances in 10 patients, three male and seven female. This lesion differs from the 'true' ureterocele which occurs with a single, normally sited ureteric orifice in that it is broader based, usually larger, more centrally situated and the ureteric orifice lies, not at the apex of the cystic protrusion, but towards its lower extremity, at the bladder neck or in the proximal urethra. The orifice may be small and stenotic, as with the true ureterocele, but more frequently it is of normal size or even larger than normal; the obstruction which causes intravesical ballooning of the lower part of the accessory ureter is due to the position of the orifice relative to the vesical sphincter rather than to its size. When the sphincter is in its resting state of tonic contraction it compresses and occludes the termination of the accessory ureter so that the ureter can only empty adequately when the sphincter relaxes during micturition.

The ipsilateral main pyelon was of normal calibre or only slightly dilated with six of the ureteroceles; in the remaining five it was considerably dilated. In four of these the corresponding ureteric orifice was normal in appearance, without, in at least two cases, vesico-ureteric reflux; there can be little doubt that the main pyelon dilatation here was the result of compressive obstruction of its intramural ureter by the ureterocele, particularly since improvement in the degree of dilatation and in radiographic function followed excision of the ureterocele in two cases (Fig. 2a and b). In one patient the twin pyelon had the features of the idiopathic megaureter.

It might be expected that an ectopic ureterocele encroaching, as it does, on the vesical outlet would interfere with micturition and lead to chronic vesical retention of urine. In fact, this occurred in only two of our patients, both male, in whom the bladder was persistently distended, trabeculated and somewhat hypertrophied. Even in these cases the obstructive effects were not particularly severe since, in both, the renal tract opposite to the ureterocele was undilated. The low incidence of dysuria with ectopic ureterocele was also recorded by Ericsson (1954) and by Stephens (1958). It is likely that the emptying and collapse of the ureterocele during micturition are responsible for this relative freedom from urethral obstruction.

The ectopic ureterocele usually leads to symptoms in early infancy; seven of our patients were less than 5 months old. These children were seriously ill with symptoms of vomiting, failure to thrive and apparent pain and all had gross pyuria. In one baby girl the ureterocele prolapsed through the urethra. Intravenous pyelography does not, as a rule, demonstrate the dilated accessory pyelon owing to depressed renal function but the ureterocele may be seen as a round filling defect within the bladder shadow. The ureterocele can usually be seen at cystoscopy, but if it is particularly large it may obscure visibility and render elucidation difficult. The ureterocele is often palpable on bimanual examination of the pelvis under anaesthesia when the bladder is empty.

The Unobstructed Idiopathic Megaureter. The unobstructed or 'idiopathic' megaureter (Stephens, 


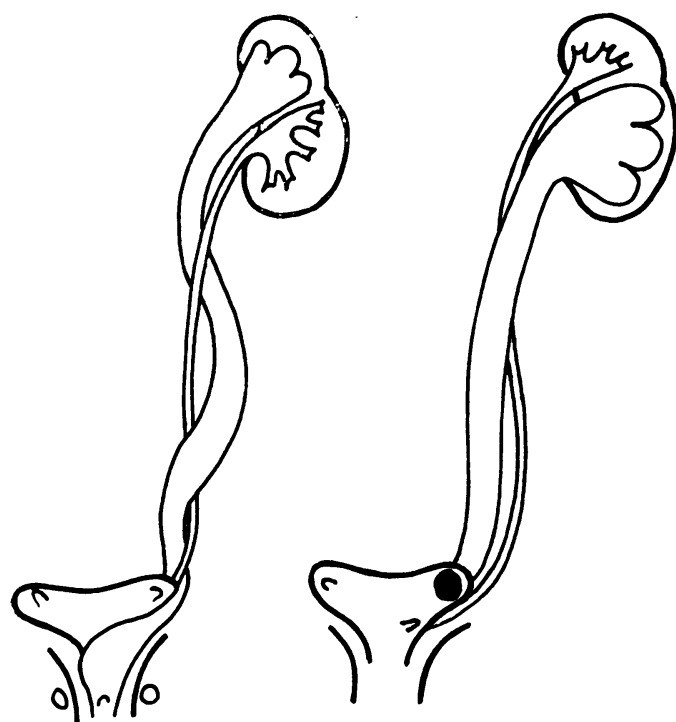

Fig. 1.-Diagram of obstructed megaureter with ectopic ureterocele. The orifice is compressed and occluded by the vesical sphincter.
1956) shows dilatation of the entire pyelon but often little or no ureteric tortuosity (Fig. 3). There is impaired concentration on pyelography. The characteristic feature of the lesion is free vesicoureteric reflux which can be demonstrated at cystography, sometimes during bladder filling, and always during micturition when the ureter and usually also the pelvis and calyces may become markedly distended with dye (Fig. 4); at the conclusion of voiding the ureter empties again into the bladder. At cystoscopy the ureteric orifice is usually seen to be widely gaping but in some cases its dilatation is only apparent during the emission of urine or when reflux occurs with increasing bladder distension. On occasion the orifice, instead of dilating, recedes from the bladder, drawing after it the adjacent bladder wall in the form of a diverticulum. The idiopathic megaureter exhibits variable degrees of dilatation and renal functional capacity, but in the individual case these features remain unaltered with the passage of time, provided infection is controlled (Stephens, 1956); the condition appears to be an inherent anomaly, the result of a developmental defect of the ureteric bud and the related renal parenchyma.

In the well-developed case the diagnosis of the idiopathic megaureter is readily established but its

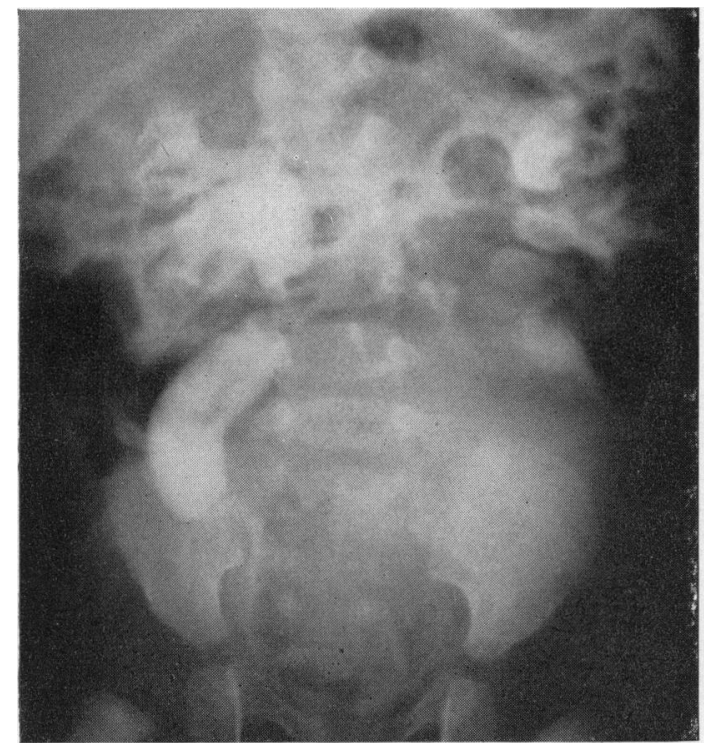

(a) Aged 2 months; before surgery. The primarily obstructed left accessory pyelon does not show. Dilated calyces of the secondarily obstructed left main pyelon are visible. The single right kidney is hydronephrotic and the lumbar ureter is dilated as a result of infection.

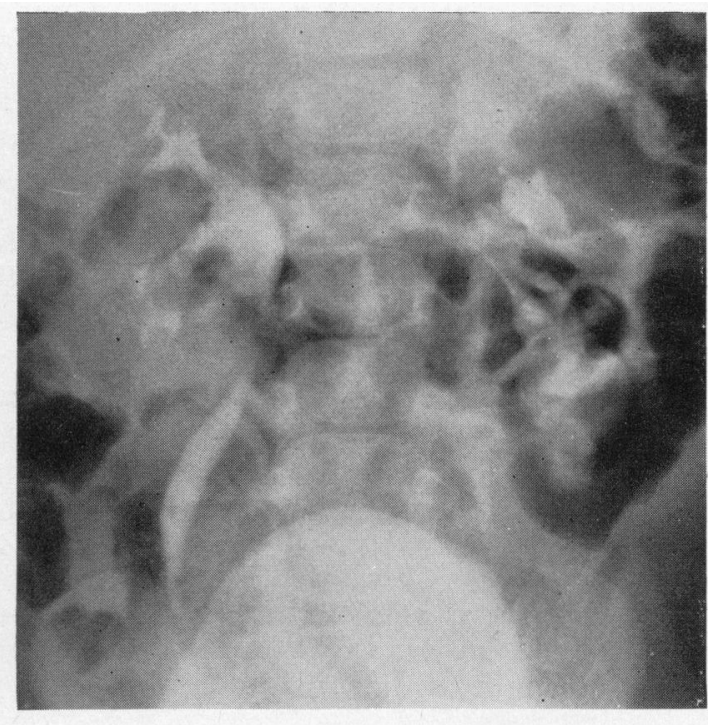

(b) Six months after excision of the ureterocele. On the left, the accessory pyelon is now visible and the main pyelon, pelvis and calyces are no longer dilated. The hydronephrosis of the right kidney has subsided; slight ureteric dilatation possibly persists.

FIG. 2.-Left ectopic ureterocele; intravenous pyelograms in infant girl. 
mildest extreme is difficult to define precisely and different observers have accepted differing diagnostic criteria. In this series only those pyelons showing fairly gross changes on intravenous pyelography and marked distension on reflux from the bladder are considered to be of this type. In some cases of complicated duplication with obvious megaureters, non-distending reflux occurred on cystography into twin or contralateral pyelons which were of normal appearance on intravenous pyelography and it was thought that, in these instances, the reflux was a temporary effect of infection. It is possible, however, that these pyelons represent the minimal degree of the idiopathic megaureter.

According to our criteria for diagnosis, idiopathic megaureter was found in seven patients, each of whom had urinary infection; in three the accessory and in four the main pyelon was involved. The affected pyelon was visible on intravenous pyelography, although with considerably diminished concentration, in three cases; in the remainder it was radiologically silent. The twin pyelon was normal in six cases; in one it was obstructed by an ectopic ureterocele. In two cases there was an ectopic ureterocele on the other side. The age at diagnosis in those patients without associated ureterocele was $3 \frac{1}{2}$ years in one and under 6 months in the remainder.

The Simple Obstructed Megaureter. One pyelon in the clinical series had the features of the 'simple' obstructed megaureter of Williams (1958) in which Murnaghan (1957) has demonstrated that the essential pathology is a functional obstruction in the undilated intramural and, in some cases, the lower extramural ureter, because of anomalous muscular patterns in the ureteric wall. In this patient, a boy of 5 years with unilateral duplication, the accessory pyelon was represented by an atretic ureter and a multicystic dysplastic kidney; the main pyelon ureter was dilated and tortuous with a normal, nonrefluxing ureteric orifice and, at operation, a short, narrow extra vesical segment. In one necropsy case of incomplete duplication the accessory pyelon was dilated but narrowed just above the point of fusion with its twin and it is possible that this megaureter had a similar pathogenesis.

The Infective Megaureter. Both the obstructed and the unobstructed megaureter may, in the young infant, be simulated on the intravenous pyelogram by the effects of urinary infection. At this age infection may lead to marked dilatation of the upper urinary tract, involving the pelvis and calyces as well as the ureter, with delay in ureteric emptying and, in some cases, vesico-ureteric reflux. This

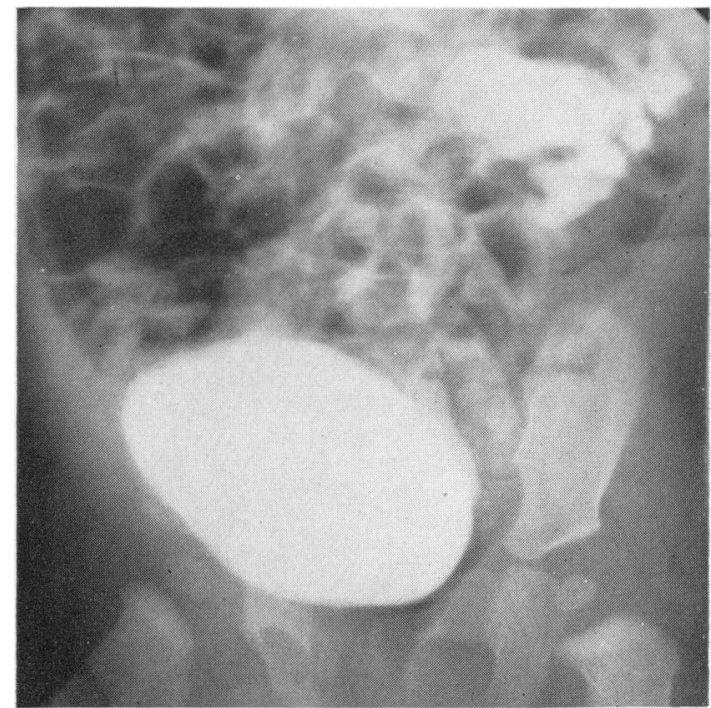

Fig. 4.-The idiopathic megaureter. Cystogram in boy aged 7 months, showing reflux into a dilated left main pyelon. This portion of the double kidney was not visible on intravenous pyelography.

infective megaureter can return to normal in respect both of dimensions and of competency of the vesicoureteric sphincter mechanism (Fig. 5a and b). Since pyuria is present with each type of lesion it may only be possible to distinguish them with certainty by repeated observation after the infection has been controlled.

Pelvic Hydronephrosis. Hydronephrosis due to obstruction at the pelvi-ureteric junction, identical with that which occurs in the single kidney, was found in three clinical cases, children aged 6,8 and 11 years, and at necropsy of a child who died with multiple abnormalities at the age of 3 days. In each case the main pyelon was affected; the absence of a recognizable pelvi-ureteric junction probably precludes the possibility of this lesion occurring in the accessory pyelon.

Radiology and Diagnosis. Radiography of complete double ureters, particularly when ureteric catheters are in position, shows localized crossing of the ureters in at least one site and sometimes in three and it is often stated that ureters obstruct one another at these points. In no case in the present series was a duplicated ureter dilated to the level of a radiological crossing. When the ureters are exposed at operation it is obvious that they lie more or less parallel to one another and that the 


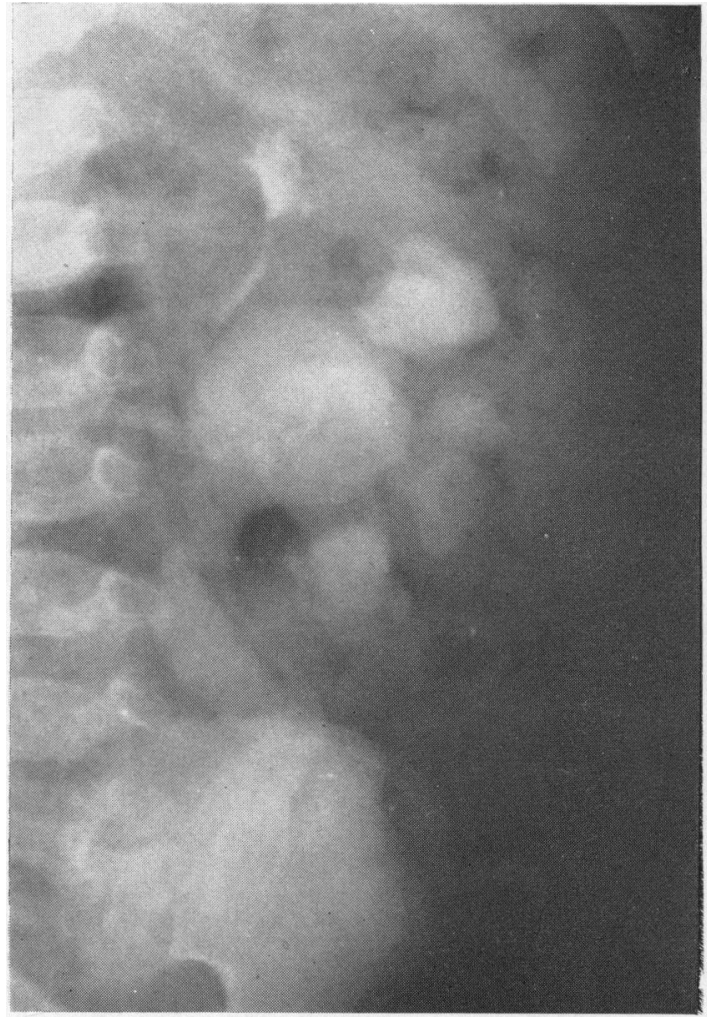

(a) Aged 2 months; hydronephrosis and ureteric dilatation affecting the main pyelon.

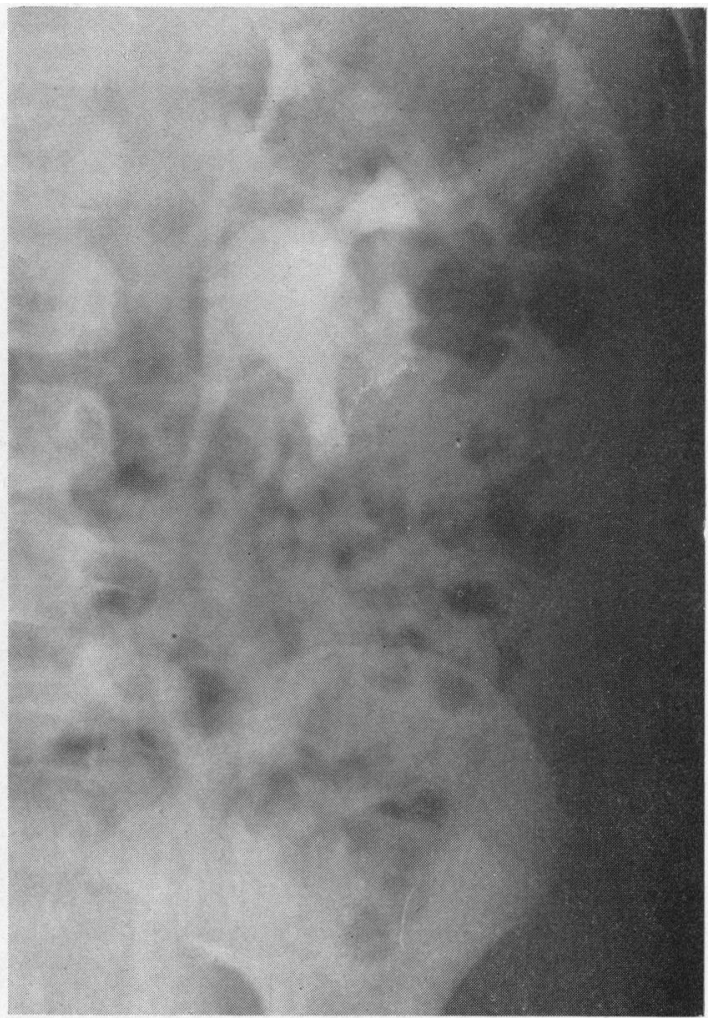

(b) Aged 3 years; the pyelon has returned to normal with the elimination of infection.

FIG. 5.-The infective megaureter; intravenous pyelograms in girl.

localized crossings are more radiologically apparent than real.

In a structurally complicated ureteric duplication there is commonly depressed function of a renal segment with radiological invisibility of the corresponding pyelon; unless the possibility of such a lesion is kept in mind in the child with chronic pyuria and cystoscopy performed, its presence may readily be overlooked. This is more likely to happen when only the larger main pyelon is apparent on intravenous pyelography. Suggestive radiological features in such cases are: an elongated renal soft tissue shadow with the visible upper calyx at a distance from the upper pole, a diminution in the number of calyces as compared with the opposite side or, since a non-visualized accessory pyelon is usually dilated, a lateral displacement of the upper pole of the pyelogram, giving the 'drooping flower' appearance or lateral and sometimes downward displacement of the pelvis and the upper ureter. Occasionally, however, the pyelogram may appear virtually normal. In the type of case with which the present review is concerned double ureteric orifices will almost certainly be seen at cystoscopy and such procedures as perirenal air insufflation, aortography or operative exploration, which are sometimes necessary for the diagnosis of the ectopic supernumerary ureter, will rarely be required.

When structural complications are present, ureteric duplication may present a highly complex picture and, especially in the young infant, it is often difficult to decide at the time of first assessment whether dilatation of a particular pyelon indicates the presence of an intrinsic obstructive or non-obstructive lesion or is secondary to disease of its twin or the result of infection. In such cases the true pathology may only become apparent by observation of the effects of conservative treatment, either surgical or medicinal.

Infection without Structural Complications. Urinary infection in association with complete 
or incomplete duplication but without other apparent anatomical anomaly of the urinary tract except, in some cases, slight ureteric dilatation, occurred in 26 children. In 12, 11 girls and one boy, the infection was transient but in 14, 13 girls and one boy, it was chronic or recurrent and, in the latter group at least, presumably associated in many cases with pyelonephritis, although this was proved histologically only in one patient. The age at presentation of these 26 patients was considerably greater than those with structural complications, the average being 6 years. Increased frequency and urgency of micturition, nocturnal enuresis of onset type and attacks of pyrexia, sometimes with loin pain, were the commonest symptoms.

The reasons for the apparent increased liability of the reduplicated but otherwise structurally normal urinary tract to the development and persistence of infection are not known with certainty. Stephens (1956), whose patients were all investigated by micturating cysto-urethrography, found that vesico-ureteric reflux and, in cases of incomplete duplication, uretero-ureteric reflux were commonly present and considered these to be important pathogenic factors. Since vesico-ureteric reflux may be the reversible result of urinary infection, distinction of cause from effect may be difficult and clinical experience suggests that reflux is of significance in the causation of infection only when the volume of regurgitated urine is large so that a large residue of urine remains after micturition. Reflux is, therefore, of great importance in respect of infection with the idiopathic megaureter in which there is a greatly increased ureteric capacity; it is unlikely that reflux into a ureter of normal calibre, even if it causes some ureteric distension, would be of sufficient quantity to be significant. Forsythe and Whelan (1958) found that vesico-ureteric reflux was commonly present in enuretic children without urinary infection. Relatively few of our patients with urinary infection and a duplicated but undilated urinary tract underwent cystography so that the incidence of ureteric reflux in these cases is not known. Reflux was, however, not present in the patient with histologically proven chronic pyelonephritis; in this 6-year-old girl the disease, obvious on intravenous pyelography because of delayed concentration, small size and approximation of calyces, was confined to the lower portion of a completely reduplicated right kidney.

Payne (1959) noted that, in patients with infection and ureteric duplication, retrograde pyelography suggested ureteric stasis as dye was still present in the pelvis or ureter 10 minutes after the injection. Image intensifier studies in five of our cases have, however, shown no delay in the emptying of undilated duplicated ureters.

The predisposition of the congenitally hypoplastic, dysplastic kidney to infection is well recognized. This anomaly, indistinguishable radiologically and macroscopically from the scarred hypotrophic kidney of chronic pyelonephritis, is identifiable histologically mainly by the presence of primitive tubules, resembling those of the foetal kidney, which have a wide lumen and a lining of deeply staining cubical cells, surrounded by a concentric arrangement of loose connective tissue which may contain angiomatous material and cartilage. The frequent association of gross dysplasia with pyelonephritis raises the question whether lesser degrees of dysplasia may predispose to the development of pyelonephritis in a kidney which appears normal radiologically. Marshall (1953) considered that persistent foetal elements in the renal parenchyma constitute loci minoris resistentiae to infection and Ericsson and Ivemark (1958), who were of the same opinion, found such elements to be present frequently with complicated ureteric duplication.

Histological material was studied from the parenchyma of 42 pyelons from the entire present series. Undoubted dysplastic tissue was found in ten. In only one of these was the corresponding ureter of normal calibre; the remainder was associated with hydronephrosis and obstructed or unobstructed megaureter or, in one instance, with an atretic ureter. In 20 specimens the renal parenchyma was histologically normal; in 15 of these the ureter was normal and in five it was dilated. Chronic pyelonephritis without dysplasia was found in 12 specimens; in one the ureter was normal and in the remainder it was pathologically dilated. Many of these last sections showed marked fibrosis and distortion of renal tissue and it was considered possible, in some instances, that dysplastic elements might have been present initially, but subsequently became obscured by scarring. In the majority of cases in which a histological specimen was obtained from a renal segment drained by a normal pelvis and ureter there were marked pathological changes in the twin pyelon; no definite conclusions can be drawn from this series, owing to the paucity of histological material from a duplicated but otherwise anatomically normal urinary tract, concerning the part played by dysplastic elements in predisposing to infection in such cases. It is apparent, however, that the renal parenchyma is frequently normal when there is no dilatation of the corresponding pyelon and that dysplasia is usually associated with megaureter and hydronephrosis, as it was in 10 of Marshall's (1953) 
12 cases. In the latter instance it is likely that the urinary stasis resulting from the dilatation of the pyelon is of greater significance in the pathogenesis of infection than the parenchymal anomaly.

The embryological sequence of events which associates a congenitally abnormal ureter with an abnormal renal parenchyma probably differs with the type of lesion. An inherently defective ureteric bud may induce the formation of a defective kidney or vice versa and it is likely that the nonprogressive idiopathic megaureter constitutes such a primary defect of germ plasm. The kidney parenchyma in three of our six cases, from which histology is available, contained dysplastic tissue and it was considered to be present possibly in a further one. With the obstructed megaureter the ureteric and renal anomalies may both be the result of obstruction; renal dysplasia is not uncommonly found with the dilated urinary tract resulting from urethral valves. A urinary obstruction present at an early period of foetal life appears to interfere with the maturation of the developing nephrons and also with the development of the ureteric musculature; this is of clinical prognostic importance in that, although some improvement in the degree of ureteric dilatation and in radiological renal function may, in some cases, follow the post-natal relief of prenatal obstruction, complete restoration to normal is never possible. The renal parenchyma from three of our six cases of ectopic ureterocele showed dysplasia and in two others its presence was thought possible; one of the specimens from the two cases of simple megaureter was dysplastic.

\section{Treatment}

The patient with ectopic ureterocele commonly presents in infancy with acute symptoms of urinary obstruction and infection and in these cases the immediate essential is the provision of free drainage of the obstructed ureter into the bladder. Gross and Clatworthy (1950) advocated, for this purpose, a small incision only in the ureterocele wall in order to prevent subsequent vesico-ureteric reflux. It has, however, been our experience, and also that of Ericsson (1954) that, both in the ectopic and the true ureterocele, simple incision does not prevent reflux and it carries the disadvantages that the small opening may diminish in size as the ureter shrinks and also that the residual redundant cyst wall may lead to later complications; in one of our patients it caused a flap valve effect at the bladder neck which interfered with micturition and necessitated a second operation. Excision of the ureterocele by open operation is therefore preferable in that it ensures drainage and carries no disadvantage as compared with incision in respect of reflux. Incision or excision of seven ectopic ureteroceles was performed in the present series. The involved pyelon showed improved function, or visibility for the first time, on intravenous pyelography in three instances (Fig. 2a and b); in all cases pyuria and ureteric dilatation persisted. Ericsson (1954) found similar radiographic improvement, but persistence of infection, in nine of 10 cases. Improved drainage of a secondarily obstructed ipsilateral main pyelon, as shown by improved radiographic function and a diminution in the degree of dilatation, resulted from decompression of the ureterocele in two of four cases (Fig. 2a and b). Some period of time must often elapse before free drainage becomes effective and the time factor may be of great importance when the contralateral renal function is defective or in cases of bilateral ectopic ureterocele. This was exemplified by one patient who required somewhat unorthodox management.

Case Report. J.S., a baby girl, was admitted to hospital at the age of 11 days with pyrexia, vomiting and diarrhoea. Her urine contained a large amount of pus and grew Esch.coli and S. viridans on culture. Intravenous pyelography showed the faint shadows of bilateral hydronephrotic kidneys. Cystoscopy revealed bilateral ectopic ureteroceles; above each ureterocele was a normal ureteric orifice. There was no reflux into any of the ureters on micturating cystography. At operation, when the child was 4 weeks old, the ureteroceles were excised by open transvesical operation; the right drained through a large orifice into the proximal urethra but no orifice could be found on the left ureterocele. The child's general condition gradually deteriorated after operation; swinging fever and repeated vomiting persisted and she failed to gain any weight. In spite of antibiotic therapy the urine was persistently infected. The blood urea rose to a maximum of $120 \mathrm{mg}$. $\%$. Intravenous pyelographysix weeks after operation showed no concentration by the right double kidney; on the left, only the main pyelon was visible, showing hydronephrosis and a dilated tortuous ureter with marked delay in emptying of the ureter into the bladder (Fig. 6a). Clinically the child appeared to empty her bladder completely. It was apparent that her failure to improve was the result of persistent impairment of drainage of the sole pyelon retaining significant function and that decompression of this pyelon was essential. Tube nephrostomy has, in our experience of such urinary obstruction, been of very temporary benefit only; the presence of an indwelling tube for more than a week or so invariably leads to the exacerbation of infection and to the patient's deterioration. A left cutaneous ureterostomy was therefore performed, both left ureters being divided just above the bladder and brought out in the left iliac fossa (Fig. 6b). The child's general condition showed an almost immediate and striking improvement 


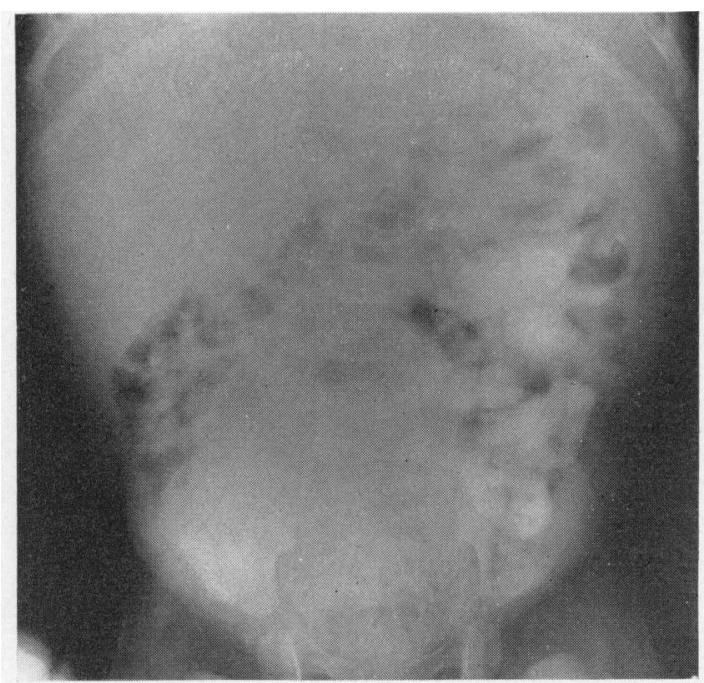

(a)

FIG. 6.-J.S., baby girl with bilateral ectopic ureteroceles.

(a) Intravenous pyelogram six weeks after excision of the ureteroceles. The right pyelons and the left accessory pyelon do not show. Impaired drainage of the left main pyelon persists. Radiograph taken 80 minutes after injection.

(b) Temporary left cutaneous ureterostomy. Both left ureters have been divided near the bladder and drain on the surface.

(c) Intravenous pyelogram three months after reimplantation of the left ureters into the bladder. Both left pyelons are visible, showing moderate dilatation. Neither right pyelon shows.

and she was discharged home two weeks later on a urinary antiseptic. Intravenous pyelography six months later again showed no dye concentration on the right side. On the left, both pyelons were now visible, each showing moderate hydronephrosis and hydroureter; a small calculus was present in the accessory ureter. The child had continued to pass urine in small quantities through the urethra. Six months after the ureterostomy had been performed the left double ureters were reimplanted into the bladder, bringing them obliquely through the bladder wall in the hope of preventing reflux, and the small mobile calculus was removed. Three months after the reimplantation, intravenous pyelography again showed no concentration by the right double kidney; both pyelons on the left showed

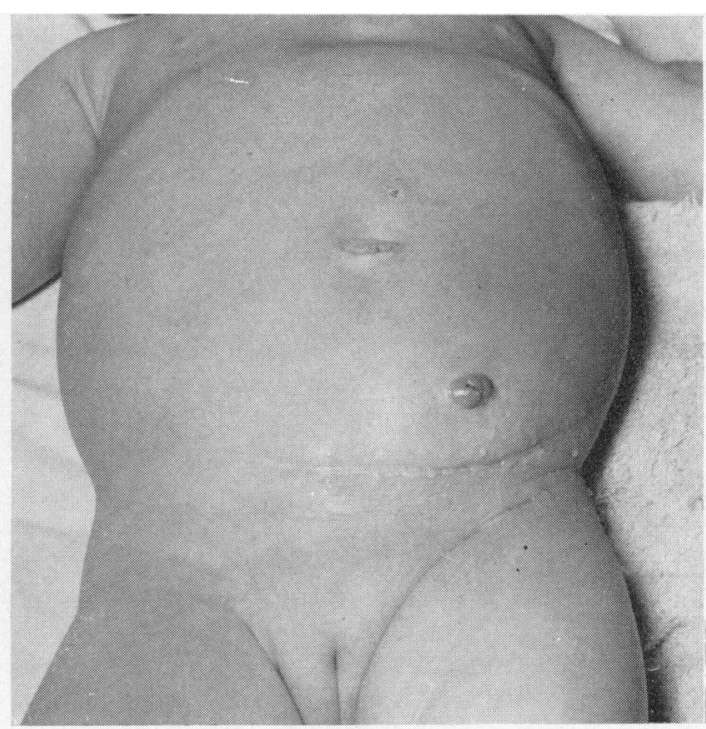

(b)

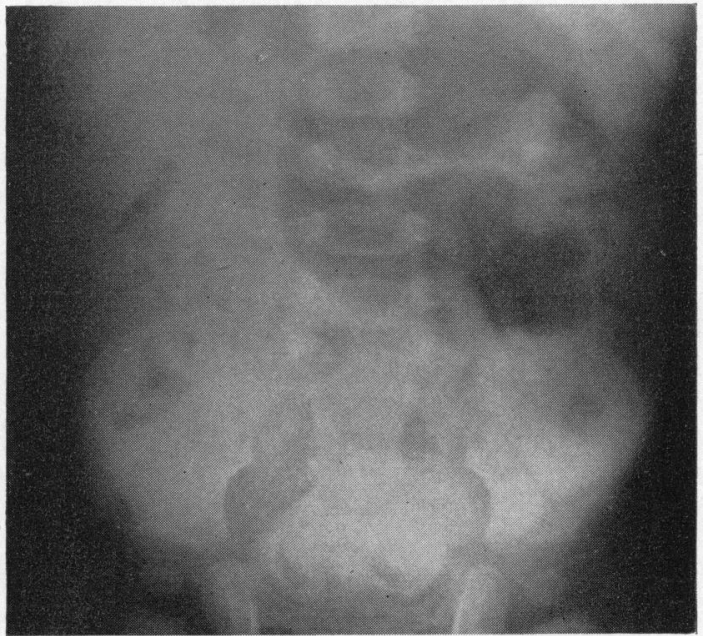

(c)

good concentration of the dye but moderate dilatation remained (Fig. 6c). Six months later the child is well and thriving. Mild pyuria and bacilluria persist in spite of prolonged chemotherapy.

Although radiographic improvement may be apparent after decompression of an ectopic ureterocele, dilatation of the accessory ureter and pyuria almost invariably persist and heminephrectomy and ureterectomy are indicated if the remainder of the urinary tract has returned to normal and the involved pyelon is thus not essential to the patient. Excisional surgery should be carried out as a primary procedure if disease is limited to the one 
pyelon and there are no secondary effects on the others. Heminephrectomy or nephrectomy was performed in seven cases in the present series.

Surgery is not required urgently with the unobstructed megaureter associated with duplication; a period of antibiotic treatment is of value, not only therapeutically, but also in allowing a more precise assessment of the pathology and, particularly in the young infant, in distinguishing intrinsic anomalies from reversible effects produced by infection. The treatment of the idiopathic megaureter depends upon its functional capacity and upon the integrity of the remainder of the urinary tract. Excision of the involved pyelon, which was performed in six of our cases, is preferable if it is dispensable because the free vesico-ureteric reflux, as with a large vesical diverticulum, leads to the accumulation of residual urine which promotes infection and endangers not only the anomalous pyelon, but also the others. When retention of the pyelon is essential the residual urine in the ureter and bladder may, in the child of co-operative age, be diminished or even eliminated by having the patient micturate every two hours and, at each occasion, empty the bladder three times at two-minute intervals (Stephens, 1956). By this means and by the prolonged use of urinary antiseptics deterioration in renal function may be prevented. Plastic operations at the uretero-vesical junction such as those described by Bischoff (1957) and by Hutch (1952), which aim at preventing vesico-ureteric reflux, have not been employed in any case in this series but they have, in our experience, been applied with success to the idiopathic megaureter involving a single pyelon; it appears likely that, with slight modification in technique, they could be used for the duplicated ureter.

Heminephrectomy presents few technical problems in cases of ureteric duplication. The line of division of the kidney may be apparent as a groove on the surface; frequently the diseased portion is firmer to palpation than the normal or is hydronephrotic so that it is nearly always possible to excise one pyelon without opening the pelvi-calyceal system of the other. It is important that the ureter is completely removed; a residual stump of ureter, not only in the case of the idiopathic megaureter but also in that of the ectopic ureterocele, particularly if the ureterocele has previously been incised or excised, acts as a diverticulum, leading to the persistence of infection and possibly, as occurred in one of our cases, to calculus formation. The double ureters often share a common wall in the lowest inch or two and, in order to avoid injury to the twin ureter or to its blood supply, only that part of the diseased ureter which is unattached to its twin must be resected, leaving the common wall intact; the resulting small aperture in the bladder at the site of the ureteric orifice requires a suture.

The simple megaureter did not present as a clinical problem in the present series. When it does so, treatment depends upon whether the involved pyelon is dispensable or must be retained. In the latter instance reimplantation of the ureter into the bladder or uretero-ureterostomy (Rothfeld, 1960) may be indicated.

The treatment of hydronephrosis due to obstruction at the pelvi-ureteric junction is the same in the duplicated kidney as in the single organ. In one of our cases pyeloplasty was performed; in the others, nephrectomy or heminephrectomy.

In the treatment of loin pain associated with uncomplicated ureteric duplication, Gutierrez (1944) recommended upper segment heminephrectomy in the adult because of presumed impaired ureteric dynamism'. This procedure has little or no justification in the child. In our five patients with loin pain the symptom was only occasional and did not appear to be severe.

The treatment of the older child with chronic or recurrent urinary infection associated with ureteric duplication, but without complicating hydronephrosis or megaureter, differs little from that of the patient without duplication; it consists mainly in the prolonged administration of urinary antiseptics and the prevention of reinfection by the avoidance of constipation, the elimination of obvious septic foci and, in the girl, local cleanliness. Gross (1953), in the belief that duplicated ureters are mutually obstructive, advocated upper segment heminephrectomy and ureterectomy in order to promote drainage from the lower and larger portion of the kidney. Unless, however, impaired ureteric drainage can be demonstrated radiologically, and this was not so in five of our cases of this type examined by the image intensifier, excisional surgery does not appear to be justifiable; its sole indication is in the unusual case in which chronic pyelonephritis can be shown to be confined to a dispensable renal segment. In the majority of children with recurrent urinary infection the attacks tend to cease at about puberty (Williams, 1958), but it must be presumed that each episode is associated with pyelonephritis and with further impairment of renal function. These patients require prolonged frequent supervision; repeated pyelographic examinations, with the object of finding indications for surgery, are of doubtful value. In our experience anomalies such as megaureter and hydronephrosis or pyelographic evidence of hypoplasia or pyelonephritis have always 
been present at the initial investigation; we have not observed them develop de novo during observation extending, in many cases, over a period of several years.

\section{Summary}

A series of 82 cases of ureteric duplication in children is described. The complications found were of two types: first, structural lesions which included ectopic ureterocele, unobstructed idiopathic megaureter, simple obstructed megaureter, infective megaureter and pelvic hydronephrosis; second, urinary infection with a duplicated but otherwise apparently structurally normal urinary tract. The pathogenesis and treatment of the various complications are discussed. Attention is drawn to the value of temporary cutaneous ureterostomy in the treatment of the patient with ectopic ureterocele and secondary obstruction of the main pyelon which persists after decompression of the ureterosele.

I am indebted to Miss Isabella Forshall and Mr. P. P. Rickham for permitting me to include their cases for the purpose of this review, and to Dr. E. G. Hall and Dr. J. Bouton for advice on the interpretation of renal histopathology.

\section{REFERENCES}

Beer, E. and Mencher, W. H. (1938). Heminephrectomy in disease of the double kidney. Report of fourteen cases. Ann. Surg., 108,705 .

Bischoff, P. (1957). Megaureter. Brit. J. Urol., 29, 416.

Braasch, W. F. (1912). The clinical diagnosis of congenital anomaly in the kidney and ureter. Ann. Surg., 56, 726.

Campbell, M. (1951). Clinical Pediatric Urology. Saunders, Philadelphia and London.

Ericsson, N. O. (1954). Ectopic ureterocele in infants and children : Clinical study. Acta Chir. Scand. Suppl., 197.

- and Ivemark, B. I. (1958). Renal dysplasia and urinary-tract infection. Ibid., 115, 58.

Forsythe, W. I. and Whelan, R. F. (1958). The occurrence and significance of vesico-ureteral reflux in children. Brit. J. Urol., 30, 189.

Gross, R. E. (1953). The Surgery of Infancy and Childhood; its Principles and Techniques. Saunders, Philadelphia and London. and Clatworthy, H. W., Jr. (1950). Ureterocele in infancy and childhood. Pediatrics, 5, 68.

Gutierrez, R. (1944). Double kidney as a source of impaired dynamism; its surgical treatment by heminephrectomy. Amer. J. Surg., $65,256$.

Hutch, J. A. (1952). Vesico-ureteral reflux in the paraplegic: cause and correction. J. Urol. (Baltimore), 68, 457.

Marshall, A. G. (1953). The persistence of foetal structures in pyelonephritic kidneys. Brit. J. Surg., 41, 38.

Murnaghan, G. F. (1957). Experimental investigation of the dynamics of the normal and dilated ureter. Brit. J. Urol., $29,403$.

Payne, R. A. (1959). Clinical significance of reduplicated kidneys. Ibid., 31, 141.

Rothfeld, S. H. (1960). Uretero-ureterostomy: A means of conservation of renal tissue. J. Urol. (Baltimore), 84, 60 .

Stephens, F. D. (1956). Double ureter in the child. Aust. N.Z. J. Surg., 26, 81.

(1958), Ureterocele in infants and children. Ibid., 27, 288. Thompson, I. M and Amar A. D. (1958). Clinical importance of ureteral duplication and ectopia. J. Amer. med. Ass., 168, 881.

Williams, D. I. (1958). Urology in Childhood. Vol. 15 (Handbuch der Urologie). Springer, Berlin. 